\title{
Treatment-related stress and coping: an important consideration in patients on hemodialysis
}

\author{
Belgüzar Kara* \\ Department of Internal Medicine Nursing, Faculty of Health Sciences, Yuksek Ihtisas University, Turkey
}

Hemodialysis (HD) is the most commonly used renal replacement therapy method in patients with end-stage renal disease worldwide, and it needs to continue throughout life unless a successful transplant can be provided [1]. However, HD is a stressful experience for both the patient and the family. This population is threatened with many potential losses and changes in life-style [2-5]. The way patients cope with treatment-related physiological and psychosocial stressors (e.g. limitation of fluid and food, fatigue, length of treatment, frequent hospital admissions, dependency on others, decrease in social life, uncertainty concerning the future, and muscle cramps) is therefore important [2]. Coping might be accepted as a mediator between treatment-related stressors and health outcomes such as depression or quality of life in patients on HD. It is reported that these patients cope better when focusing on the problem with controllable stressors and on the emotion with less-controllable stressors $[2,6,7]$.

There are many studies on the treatment-related stressors experienced and coping strategies used by patients on HD [2,8-12]. In $\mathrm{Tu}$ et al. [8]'s study, Taiwanese patients on HD aged 20-45 years were found to have high stress levels. Moreover, patients reported having more physiological stressors than psychosocial stressors and using more problem-focused coping strategies than emotion-focused ones [8]. On the other hand, in Parvan et al. [9]'s study, most Iranian patients on HD reported using emotion-focused coping strategies more than problem-focused strategies. In a recent cross-sectional study of 218 Jordanian patients on HD, Musa et al. [10] reported that patients demonstrated mild to moderate stress. The investigators have also showed that patients on HD used religious and spiritual beliefs as coping strategies to overcome their stress [10]. Ramirez et al. [11] suggested that while religious struggle was associated with greater psychological distress and impaired health-related quality of life, positive religious coping was associated with improved quality of life among Brazilian patients on HD. In Kara [2]'s study, higher treatmentrelated stress levels were also associated with lower coping strategies in Turkish patients on HD [2]. Interestingly, in the Japan Dialysis Outcomes and Practice Patterns Study of 1,354 patients on HD, it was found that "problem-focused engagement" as coping strategies were associated with both longer survival and improvement in physical functioning and mental health [12].

Consequently, the results of studies showed that patients on HD often experience high stress levels, and use both emotion-focused and

Copyright: (C2018 Kara B. This is an open-access article distributed under the terms of the Creative Commons Attribution License, which permits unrestricted use, distribution, and reproduction in any medium, provided the original author and source are credited. problem-focused coping strategies [2,8-12]. In this context, nurses play an integral role in providing care, symptom control, psychosocial support, stress management and advice for patients on $\mathrm{HD}$ and their family members. Nurses need to define the stressors related to treatment in patients on HD and the coping mechanisms and take these into consideration during care. Moreover, the scientific data regarding the treatment-related stressors and coping strategies in patients on HD were recognized. Future research will enhance understanding of treatment-related stressors and coping strategies in patients on $\mathrm{HD}$ and develop more effective interventions [1-7].

\section{References}

1. Kara B (2016) The importance of fluid management in patients on hemodialysis: An overview. Int $J$ Nurs Papers 1: 1-2.

2. Kara B (2006) A validity and reliability study of the Hemodialysis Stressor Scale. Zonguldak Karaelmas University Zonguldak School of Health. J Health Education Research 2: 64-71 (in Turkish).

3. Kara B, Caglar K, Kilic S (2007) Nonadherence with diet and fluid restrictions and perceived social support in patients receiving hemodialysis. J Nurs Scholarship 39: 243-248.

4. Kara B (2016) Determinants of thirst distress in patients on hemodialysis. Int Urol Nephrol 48: 1525-1532.

5. Kara B (2018) Health beliefs related to salt-restricted diet and associated factors in Turkish patients on hemodialysis. J Transcult Nurs 29: 155-164.

6. Kara B, Akbayrak N (2005) Hemodialysis: How can patients learn to cope? Journal of Health and Society 15: 3-7 (in Turkish)

7. Kara B, Akbayrak N (2005) Life with hemodialysis: Stress and coping. Journal of Health and Society 15: 12-17 (in Turkish).

8. Tu HY, Shao JH, Wu FJ, Chen SH, Chuang YH (2014) Stressors and coping strategies of 20-45-year-old hemodialysis patients. Collegian 21: 185-192.

9. Parvan K, Ahangar R, Hosseini FA, Abdollahzadeh F, Ghojazadeh M, et al. (2015) Coping methods to stress among patients on hemodialysis and peritoneal dialysis. Saudi J Kidney Dis Transpl 26: 255-262.

10. Musa AS, Pevalin DJ, Al Khalaileh MAA (2017) Spiritual well-being, depression, and stress among hemodialysis patients in Jordan. J Holist Nurs. [in press].

11. Ramirez SP, Macêdo DS, Sales PM, Figueiredo SM, Daher EF, et al. (2012) The relationship between religious coping, psychological distress and quality of life in hemodialysis patients. J Psychosom Res 72: 129-135.

12. Niihata K, Fukuma S, Akizawa T, Fukuhara S (2017) Association of coping strategie with mortality and health-related quality of life in hemodialysis patients: The Japan Dialysis Outcomes and Practice Patterns Study. PLoS One 12: e0180498.
${ }^{\star}$ Correspondence to: Belgüzar Kara, Professor, Department of Internal Medicine Nursing, Faculty of Health Sciences, Yuksek Ihtisas University, Turkey, E-mail: belguzarkara@yiu.edu.tr

Received: February 28, 2018; Accepted: March 19, 2018; Published: March 22, 2018 